\title{
¿CUÁNDO DECIMOS QUE UN DIAGRAMA ES UN DIAGRAMA LÓGICO? UN ESTUDIO COMPARATIVO
}

\section{WHEN DO WE SAY THAT A DIAGRAM IS A LOGIC DIAGRAM? A COMPARATIVE STUDY}

\author{
$*$ \\ J.-Martín Castro-Manzano \\ Profesor Investigador \\ Facultad de Filosofía \\ UPAEP Universidad21 sur 1103 , \\ Barrio de Santiago, C.P. 72410 \\ Puebla, Puebla, México \\ josemartin.castrolupaep.mx
}

Aunque las ventajas representativas de los diagramas han sido ampliamente reconocidas, sus virtudes inferenciales no siempre han recibido la misma atención. Existe una tradición que sostiene que el razonamiento basado en pruebas lingüísticas es esencial en lógica y matemáticas, pero que el razonamiento basado en diagramas no lo es; sin embargo, gracias a ciertos proyectos de investigación sobre razonamiento diagramático, hoy tenemos modelos que nos ayudan a comprender de manera más precisa los conceptos de diagrama lógico e inferencia diagramática. Siguiendo algunos de los planteamientos de estos modelos, en esta contribución llevamos a cabo un estudio comparativo de diez sistemas diagramáticos en términos lógicos y representativos con el propósito de ofrecer una respuesta a la pregunta de qué es lo que hace que un diagrama sea un diagrama lógico. Nuestra respuesta preliminar es que un diagrama lógico es un diagrama dentro de un sistema diagramático que es correcto y completo con respecto a una clase de inferencias válidas dada una base deductiva. Esta definición parece ser adecuada porque nos permite incluir a aquellos sistemas de diagramas que, por sus propiedades, típicamente consideramos como diagramas lógicos bona fide, pero excluye diagramas que no solo son incorrectos o incompletos, sino aquellos que ni siquiera son inferenciales.

Palabras clave: Razonamiento diagramático, silogística, historia de la lógica. 


\section{2 - Cogency, Journal of reasoning and argumentation}

Although the representative advantages of diagrams have been widely recognized, their inferential virtues have not always received the same attention. There is a tradition that holds that reasoning based on linguistic proofs is essential in logic and mathematics, but that reasoning based on diagrams is not; however, thanks to some research projects on diagrammatic reasoning, today we have models that help us understand the concepts of logic diagram and diagrammatic inference with more precision. By following the tenets of these models, in this contribution we perform a comparative study of ten diagrammatic systems in logical and representative terms with the purpose of offering an answer to the question of what makes a diagram a logic diagram. Our preliminary answer is that a logic diagram is a diagram within a diagrammatic system that is correct and complete with respect to a class of valid inferences given a deductive base. This definition seems to be adequate because it allows us to include those systems of diagrams that, due to their properties, we typically consider as bona fide logic diagrams, but it excludes diagrams that are not only incorrect or incomplete, but those that are not even inferential.

Keywords: Diagrammatic reasoning, syllogistic, history of logic. 


\section{Introducción}

¿Qué distingue a un diagrama lógico de otros diagramas? Consideremos, a modo de ejemplo, un diagrama de flujo, un diagrama de Gantt y un diagrama de Euler. Seguramente podemos reconocer que, aunque todos estos diagramas comparten ciertas características visuales o espaciales, también se distinguen porque difieren, entre otros atributos, por sus usos y sus objetivos: un diagrama de flujo, típicamente, pretende representar un algoritmo; un diagrama de Gantt se usa para planificar un proyecto; y un diagrama de Euler, usualmente, busca modelar un argumento.

Ahora bien, como esta respuesta puede dar cuenta de la diferencia entre diferentes tipos de diagramas, uno podría pensar que lo que distingue a un diagrama lógico de otros diagramas es, precisamente, su función inferencial. Esto parece ser verdad, pero puesta así, una respuesta como esta carece de profundidad, pues no explica en qué consiste dicha función. Una pregunta más interesante, en consecuencia, podría ser la siguiente: ¿Cuándo podemos decir que un diagrama es un diagrama lógico? O en otras palabras, ¿cuándo podemos decir que un diagrama tiene dicha función inferencial?

La literatura consolidada, iniciando con (Shin, 1994; Allwein y Barwise, 1996), nos ofrece varios modelos y teorías que nos ayudan a representar y a comprender de manera más clara y precisa los conceptos de diagrama lógico e inferencia diagramática, por lo que nos puede ayudar a responder la pregunta anterior; y así, con el propósito de ofrecer una respuesta más sistemática a nuestra pregunta inicial, en este estudio llevamos a cabo un estudio comparativo de diez sistemas diagramáticos en términos inferenciales (cfr. Larkin y Simon, 1987) y representativos (cfr. Nakatsu, 2010) asumiendo los criterios generales de la literatura usual.

Para llevar a cabo este estudio, que bien podría considerarse como una nota técnica al pie de una historia de los diagramas lógicos (cfr. Moktefi y Shin, 2013), primero ofrecemos algunas observaciones preliminares sobre el estado de la cuestión así como una breve descripción de la noción de inferencia diagramática (\$2). Asumiendo esas observaciones preliminares, proponemos una metodología y procedemos a realizar un análisis comparativo $(\$ 3)$; y finalmente, para terminar, concluimos con algunas consideraciones sumatorias $(\$ 4)$.

\section{Preliminares}

\subsection{La tradición}

Si bien los diagramas han sido tradicionalmente considerados como herramientas heurísticas de representación en lugar de portadores de inferencia, este aspecto viene siendo cuestionado desde ya hace varias décadas (Gardner, 1958; Swoyer, 1991; Shin, 1994; Chandrasekaran, Glasgow y Narayanan, 1995; Stenning y Oberlander, 1995; Allwein y 
Barwise, 1996; Nakatsu, 2010; Moktefi y Shin, 2013). En efecto, ejemplos notables en la historia de la ciencia, como veremos en la siguiente sección, sugieren el reconocimiento de sus cualidades representativas; sin embargo, cuando se trata de la naturaleza lógica de los diagramas, existe una tradición que sostiene que el razonamiento basado en pruebas lingüísticas es esencial en lógica y matemáticas, pero el razonamiento basado en diagramas, sin importar qué tan útil (cfr. Nelsen, 1993) o elegante (cfr. Polster, 2004) pueda ser, no lo es, puesto que no es razonamiento bona fide.

Lagrange, por ejemplo, enfatizó en el Avertissement a la primera edición de su Mécanique Analytique que no haría uso de figuras en su obra (el énfasis es nuestro):

On ne trouvera point de Figures dans cet Ouvrage. Les méthodes que j’y expose ne demandent ni constructions, ni raisonnemens géométriques ou méchaniques, mais seulement des opérations algébriques, assujetties à une marche réguliere et uniforme. (Lagrange, Boissonnade y Vagliente, 1989, p. vi)

En algún momento, Leibniz compartió una opinión similar (el énfasis es nuestro):

La force de la démonstration est indepéndante de la figure tracée, qui n'est que pour faciliter l'intelligence de ce qu'on veut dire et fixer l'attention; ce sont les propositions universelles, c’estàdire les définitions, les axiomes et les théorèmes déjà démontrés qui font le raisonnement et le soutiendraient quand la figure n'y serait pas. (Leibniz, Remnant y Bennett, 1996, p. 309)

Dieudonné, en la tradición de la Bourbaki, impulsó una adherencia estricta a los métodos axiomáticos sin apelar a la intuición geométrica, por lo menos en las pruebas formales (el énfasis es nuestro):

Although this volume includes considerable material generally treated in more elementary courses (including what is usually called "Advanced Calculus") the point of view from which this material is considered is completely different from the treatment it usually receives in these courses. [...] This has also as a consequence the necessity of a strict adherence to axiomatic methods, with no appeal whatsoever to "geometric intuition", at least in the formal proofs: a necessity which we have emphazised deliberately abstaining from introducing any diagram in the book. My opinion is that the graduate student of today must, as soon as possible, get a thorough training in this abstract and axiomatic way of thinking, if he is ever to understand what is currently going on in mathematical research. (Dieudonné, 1996, p.v) 
Neil Tennant, también, alguna vez sugirió que un diagrama es solo una heurística para incitar ciertos patrones de inferencia (el énfasis es nuestro):

When doing geometric proofs, one often draws diagrams as an aid to the imagination both in finding proofs and in following them. One draws, say, 'triangle $\mathrm{ABC}$ ' on the page; one constructs lines parallel to its sides and passing through opposite vertices; one drops lines from vertices perpendicular to the other sides or the continuations thereof; one draws angle bisectors, or lines from the vertices to the midpoints of the other sides, and so on. It is now commonplace to observe that the diagram stands for no particular triangle; that it is only an heuristic to prompt certain trains of inference; that it is dispensable as a proof-theoretic device; indeed, that is has no proper place in the proof as such. For the proof is a syntactic object consisting only of sentences arranged in a finite and inspectable array. (Tennant, 1986, pp. 303-304)

Y un tratamiento similar se extiende, por ejemplo, en los libros y manuales sobre técnicas de demostración (el énfasis es nuestro):

In thinking about sets, it is sometimes helpful to draw diagrams that illustrate how they combine under various set operations. In doing this we often represent a set with a circle (or oval), which we regard as enclosing all the elements of the set. Such diagrams can illustrate how sets combine using various operations. [...] Such graphical representation of sets are called Venn diagrams, after their inventor, British logician John Venn, 1834-1923. Though you are not likely to draw Venn diagrams as a part of a proof of any theorem, you will probably find them to be useful "scratch work" devices that help you to understand how sets combine, and to develop strategies for proving certain theorems or solving certain problems. (Hammack, 2013, pp. 20-21)

Este sesgo en contra del razonamiento basado en diagramas se puede resumir en dos objeciones generales: que los diagramas, por sus características espaciales, inducen errores inferenciales y que no son susceptibles de generalización. Consideremos, a este efecto, una de las "falacias visuales" más populares en la Figura 1.

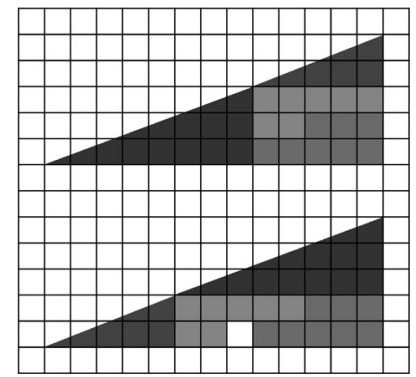

Figura 1. El cuadro perdido (cfr. Gardner, 1956) 
En esta "ilusión óptica” parece que podemos convertir el triángulo superior (i.e. la premisa) en el triángulo inferior (i.e. la conclusión) mediante un simple reacomodo de las piezas del primero, pero cuando obtenemos el segundo triángulo parece que una pieza ha desaparecido. El diagrama, por tanto, parece inducirnos a error, por lo que contraviene un principio básico de conservación de información.

Por otro lado, si consideramos, por ejemplo, el caso de la "prueba visual" del Teorema de Pitágoras (Fig. 2), parece que tenemos otro tipo de problema: que dicho resultado no puede generalizarse, pues el diagrama ofrece un resultado particular y concreto, a saber, que la prueba funciona para ese diagrama de la Figura 2 y no necesariamente para cualquier otro, lo cual parece contravenir un principio básico de universalización de información.

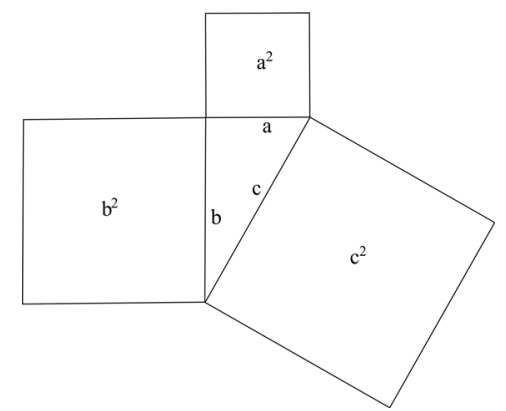

Figura 2. Una prueba visual del Teorema de Pitágoras

No obstante, no vemos cómo es que estas objeciones son de tipo knock-out, ni siquiera knock-down. Para empezar, podemos rastrear una suerte de respuesta a las objeciones anteriores en una distinción elemental que Newton ofreció en el prefacio a la primera edición de su Principia Mathematica (el énfasis es nuestro):

\footnotetext{
But as artificers do not work with perfect accuracy, it comes to pass that mechanics is so distinguished from geometry that what is perfectly accurate is called geometrical; what is less so, is called mechanical. However, the errors are not in the art, but in the artificers. He that works with less accuracy is an imperfect mechanic; and if any could work with perfect accuracy, he would be the most perfect mechanic of all, for the description of right lines and circles, upon which geometry is founded, belongs to mechanics. (Newton, 1979, p. 11)
}

Newton parece sugerir que el razonamiento basado en pruebas lingüísticas se puede reducir a razonamiento mecánico, y al proponer esta reducción ofrece una distinción que muestra que las objeciones que se pueden avanzar en contra del razonamiento diagramático son también objeciones en contra del razonamiento basado en pruebas lingüísticas, pues en este tipo de pruebas también ocurren-y quizás en mayor medida-errores (las típicas falacias 
son un buen ejemplo de errores que contravienen la preservación de información) y las proposiciones no siempre son susceptibles de generalización (algunas hipótesis y conjeturas pueden ser un ejemplo de objetos proposicionales que contravienen un principio de universalización). Pero si las objeciones en contra del razonamiento diagramático también se pueden aplicar en contra del razonamiento basado en pruebas lingüísticas, entonces el error y la incapacidad de generalización no son esenciales o propios de las representaciones diagramáticas - o con toda justicia, de las representaciones lingüísticas-sino, como quizá diría Newton, de los agentes que razonan: el problema, tal vez, no está tanto en el arte como en el artífice.

\subsection{Lógica y diagramas}

Así pues, si el rechazo a los diagramas como fuentes genuinas de inferencia se basa en las objeciones anteriores, no vemos cómo es posible no construir un caso similar para rechazar también las pruebas lingüísticas. Pero por otro lado, si la lógica tiene como objetivo estudiar la relación de inferencia y esta se estudia a través de sistemas definidos mediante elementos sintácticos y semánticos, tampoco vemos algún argumento convincente que justifique que la sintaxis o la semántica tengan que estar intrínsecamente asociadas a un único tipo de representación, en especial, a un tipo de representación lingüística. Moktefi y Shin argumentan:

\footnotetext{
All syntax does is to stipulate vocabulary, well-formed units, and a manual of permissible transformations. Semantics defines a function-style match from syntactic elements with non-syntactic entities so that ambiguity is avoided. Therefore, we believe diagrammatic logical systems are possible in principle. (Moktefi y Shin 2012, p. 612)
}

En efecto, para representar información sintáctica o semántica podemos usar diferentes tipos de representaciones: internas o externas (Larkin y Simon, 1987). Típicamente, las internas incluyen imágenes mentales, por ejemplo (cfr. Johnson-Laird, 2012); mientras que las externas incluyen, entre otros, objetos físicos sobre papel, pizarrones o monitores. Las representaciones externas todavía pueden dividirse en dos grupos: lingüísticas y diagramáticas (Larkin y Simon, 1987). Las representaciones lingüísticas, como su nombre sugiere, son secuencias de proposiciones en un lenguaje particular. Las representaciones diagramáticas, por otro lado, son secuencias de diagramas que contienen información en un locus determinado, incluyendo información sobre las relaciones de los loci adyacentes: los diagramas, pues, son gráficos que indizan información mediante su ubicación en un plano (Larkin y Simon, 1987; Nakatsu, 2010). La principal diferencia entre las representaciones diagramáticas y las lingüísticas es que, debido a su naturaleza espacial, las primeras 
preservan información explícita sobre relaciones topológicas, mientras que las segundas no lo hacen-si bien pueden, por supuesto, expresar otro tipo de relaciones. Esta naturaleza espacial les confiere algunas ventajas computacionales: los diagramas agrupan información simultáneamente, de tal suerte que evitan largos procesos de búsqueda y garantizan la posibilidad de aplicar restricciones operacionales (ya sea en términos de free rides (Shimojima, 1996), interpretaciones directas (Stenning, 2000), o iconicidad operacional (Stjernfelt 2011)) para permitir la automatización de inferencias perceptuales (Larkin y Simon, 1987).

Entonces, si asumimos que el razonamiento es un proceso que produce información (i.e. una conclusión) dada información previa (i.e. unas premisas) - siguiendo ciertas normas, por supuesto-y la información puede ser representada diagramáticamente, no resulta incómodo sugerir que la inferencia diagramática es la unidad de medida del razonamiento diagramático: una inferencia diagramática sería (in)correcta dependiendo del (in)cumplimiento de ciertas normas. Esta relación de inferencia definiría nuestras intuiciones sobre las nociones informales de inferencia visual o argumento visual y seguiría, ex hypothesi, normas estructurales clásicas (digamos, reflexividad, monotonicidad y corte), por ejemplo, a través funciones inferenciales de tipo free ride, esto es, a través de procesos mediante los cuales un agente produce información sin seguir pasos específicamente diseñados para producirla, i.e., procesos que le permiten alcanzar automáticamente (y algunas veces hasta inconscientemente) una representación diagramática de una conclusión a partir de la representación diagramática de unas premisas (Shimojima, 1996, p. 32).

Esta última consideración es de suma importancia para nuestro estudio porque nos permite ofrecer un criterio para distinguir varios tipos de diagramas por sus funciones. Para ilustrar la existencia de un criterio de este tipo basta con hacer un breve recorrido histórico del uso de diagramas. Por ejemplo, los Cuadrados de Oposición, usualmente atribuidos a Apuleyo (Londey y Johanson, 1987, p. 109) o los diagramas para silogismos imputados a Amonio Hermiae o a Juan Filópono (Hamilton, Mansel y Veitch, 1865, p. 420), son diagramas que pretenden llevar a cabo funciones inferenciales. Pero también podemos rastrear diagramas con otras funciones.

Ars Magna de Llull (1501), por ejemplo, incluye dispositivos diagramáticos cuyo objetivo es explicar la naturaleza divina a aquellos incapaces de entenderla, como si los métodos diagramáticos fueran más convincentes o expresivos que las representaciones proposicionales (Fig. 3a). Thomas Murner (1509) usó imágenes en su Logica Memorativa con la finalidad de enseñar lógica (Murner, 2017) (Fig. 3b). Simon Stevin desarrolló un diagrama (fascinante) para ilustrar su demostración de que la eficiencia del plano inclinado es una consecuencia lógica de la imposibilidad del movimiento perpetuo (Stevin, 1586) (Fig. 3c). Descartes, al igual que otros pensadores renacentistas como Kepler (Kepler, Aiton, Duncan y Field, 1997) 
(Fig. 3d), hizo buen uso de diagramas para modelar hipótesis, como la de la glándula pineal (Descartes, Miller y Miller, 1983) (Fig. 3e). Incluso Kant, en colaboración con Jäsche, parece haber desarrollado un tipo de diagramas regionales en la Jäsche Logik (Kant y Young, 1992) con el propósito de explicar la teoría general de la lógica (Fig. 3f). Y, por supuesto, además de los ejemplos científicos como los diagramas anatómicos de Vesalio en De Humani Corporis Fabrica (Fig. 3g) o los modelos Ptolemaico (1586) (Fig. 3h) y Copernicano (1543) (Fig. 3i), tenemos diagramas ingenieriles y literarios, como el mapa del infierno de Dante (Fig. 3j) (s. XIX), el escusado de Harington (Fig. 3k) (1596) o los emoticones-en el número 212-de la revista Puck (Fig. 31) (1881).

Todos estos ejemplos muestran cómo es que las ventajas representativas de los diagramas han sido ampliamente reconocidas a lo largo de la historia, quizá por las facilidades computacionales que otorgan al explicar, enseñar, ilustrar o modelar ideas. Y como estas funciones son ubicuas en diferentes ciencias y disciplinas (cfr. Perini 2012; Nakatsu, 2010) no sorprende que, al día de hoy, los diagramas sigan gozando de amplia aceptación en términos representativos; sin embargo, los ejemplos anteriores no necesariamente son casos de diagramas lógicos o inferenciales.

Según una definición de Gardner (1958, p. 28), los diagramas lógicos son figuras geométricas bidimensionales que son isomorfas a la estructura de proposiciones lógicas. Aunque esta definición es una buena aproximación inicial, se centra únicamente en la estructura interna de las proposiciones y pierde de vista que los diagramas lógicos deberían estar definidos por una función inferencial distinta de una función explicativa, didáctica, ilustrativa $o$ modeladora: los diagramas lógicos deberían ser, pues, figuras geométricas bidimensionales que implementan funciones inferenciales. Nuestro estudio tiene como propósito explorar esta hipótesis asumiendo la herramienta conceptual del free ride. 
80 - Cogency, Journal of reasoning and argumentation

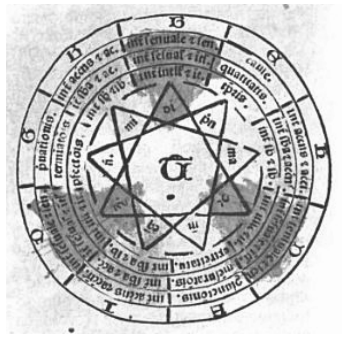

(a)

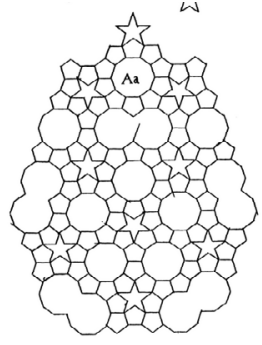

(d)



$(\mathrm{g})$

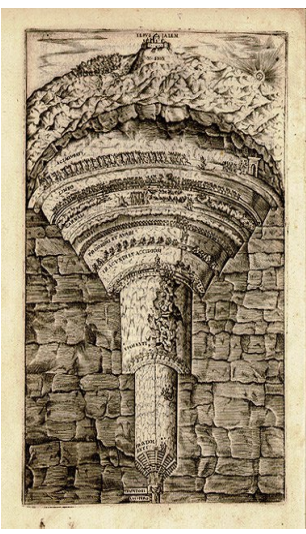

(j)

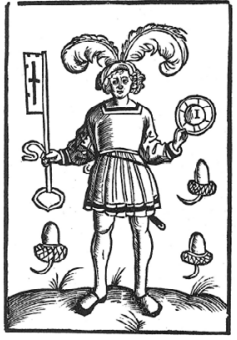

(b)

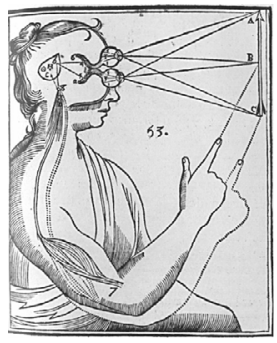

(e)

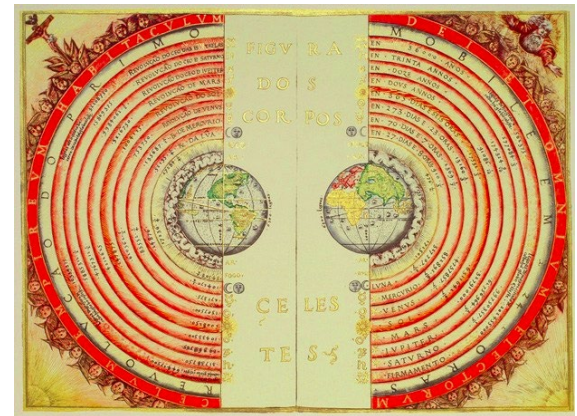

(h)

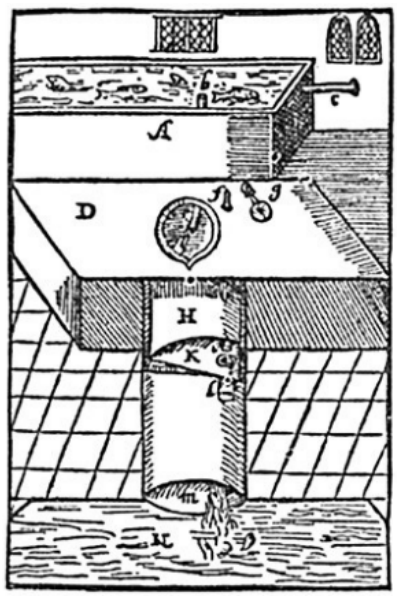

(k)

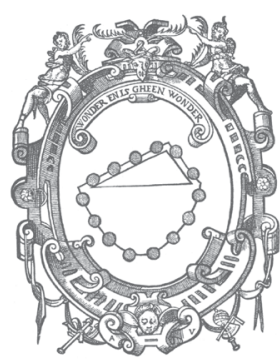

(c)



(f)

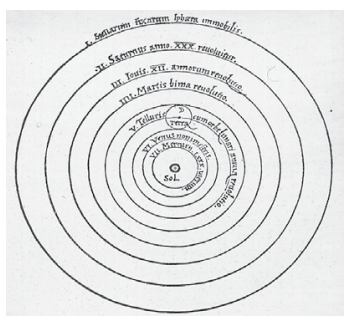

(i)



(1)

Figura 3. Algunos ejemplos de diagramas 


\section{Estudio comparativo}

Así pues, aunque hay una gran variedad de tipos de diagrama, en este trabajo restringimos nuestro estudio a aquellos diagramas que pretenden ser lógicos o inferenciales. En lo que sigue exponemos el diseño metodológico para llevar a cabo nuestro estudio.

\subsection{Diseño metodológico}

En primer lugar, definimos una base deductiva con el objetivo de normalizar los sistemas a comparar. Para este fin utilizamos la silogística porque, por su simplicidad, parsimonia y amplitud, nos ofrece una teoría lógica deductiva típica y poco controversial, además de que es el sistema lógico subyacente a la mayoría de los sistemas que estudiamos. Y para simplificar el análisis, asumimos lo que llamamos Lema de Aristóteles (Pr. An. A1, 25b1) —a saber, que todos los silogismos válidos pueden reducirse a silogismos válidos de la primera figura (vide Sección 3.2). Posteriormente, ofrecemos un conjunto de definiciones operacionales para delimitar los aspectos lógicos y representativos de nuestro interés. En particular, los aspectos lógicos que nos interesan incluyen los conceptos de corrección y completitud; mientras que los aspectos representativos incluyen los conceptos de comprensión y claridad (Nakatsu, 2010). Posteriormente, tomamos cada sistema diagramático y exponemos sus elementos básicos a partir de una fuente principal—aunque ciertamente no la única-para, finalmente, evaluar su función inferencial mediante la representación de los silogismos de la primera figura.

\subsection{Base deductiva}

La silogística es una lógica de términos que tiene sus orígenes en los Primeros Analíticos y que estudia la relación de inferencia entre proposiciones categóricas. Una proposición categórica es una proposición compuesta por dos términos, una cantidad y una cualidad. El sujeto y el predicado de la proposición se llaman términos: el término-esquema $S$ denota el término sujeto de la proposición y el término-esquema $\mathrm{P}$ denota el predicado. La cantidad puede ser universal (Todo) o particular (Algún) y la cualidad puede ser afirmativa (es) o negativa (no es). Estas proposiciones categóricas se denotan mediante una etiqueta ( $a$ (para la universal afirmativa, $\mathrm{S} a \mathrm{P}$ ), $e$ (para la universal negativa, $\mathrm{S} e \mathrm{P}$ ), $i$ (para la particular afirmativa, $\mathrm{Si}$ P), yo (para la particular negativa, $\mathrm{SoP}$ )) que nos permite determinar una secuencia de tres proposiciones que se conoce como modo. Un silogismo categórico, entonces, es un modo ordenado de tal manera que dos proposiciones fungen como premisas y la última como conclusión. Al interior de las premisas existe un término que ocurre en ambas premisas pero no en la conclusión: este término especial, usualmente denotado con el término-esquema $\mathrm{M}$, funciona como un enlace entre los términos restantes y es conocido como término medio. De acuerdo a la posición del término medio se pueden definir 
cuatro arreglos o figuras que codifican los modos o patrones silogísticos válidos (Cuadro 1).1 De estas cuatro figuras, consideraremos únicamente los silogismos de válidos de la primera figura.

\begin{tabular}{|c|c|c|c|}
\hline PRIMERA FIGURA & SEGUNDA FIGURA & TERCERA FIGURA & CUARTA FIGURA \\
\hline aaa & eae & iai & aee \\
$e a e$ & aee & aii & iai \\
aii & eio & oao & eio \\
eio & aoo & eio & \\
\hline
\end{tabular}

Cuadro 1. Silogismos válidos

\subsection{Definiciones operacionales}

Los aspectos lógicos y representativos que nos interesan son cuatro: corrección y completitud, y comprensión y claridad. Operacionalmente, decimos que un sistema de diagramas es correcto si y solo si cuando produce free rides, configura (i.e. dibuja) silogismos válidos. Para evaluar la corrección de un sistema dibujamos los free rides de la primera figura y revisamos si corresponden a silogismos válidos. Decimos que un sistema de diagramas es completo si y solo si produce free rides cuando configura silogismos válidos. Para evaluar la completitud de un sistema ofrecemos silogismos válidos y revisamos si configuran free rides. 2

Decimos que un sistema de diagramas es comprensivo si y solo si permite entender visualmente el concepto de función inferencial. Para evaluar la comprensión de un sistema revisamos los free rides disponibles y observamos la siguiente regla: si el free ride se lee de manera directa y sin necesidad de apelar a explicaciones lingüísticas adicionales, decimos que el sistema ofrece comprensión. Por último, decimos que un sistema de diagramas es claro si y solo si tiene un vocabulario bien definido. Para evaluar esta última propiedad observamos la siguiente regla: todo objeto diagramático debe estar originalmente definido y cada ocurrencia de dicho objeto representa lo que originalmente pretende representar.

\subsection{Sistemas diagramáticos}

Los sistemas que hemos elegido para este estudio están explícitamente relacionados con la silogística (i.e. nuestra base deductiva) y son los siguientes: los diagramas lineales de Averroes, los diagramas lineales de Leibniz, los diagramas lineales de Lambert, los dia-

\footnotetext{
1 Por mor de brevedad, pero sin pérdida de generalidad, omitimos los silogismos que requieren carga existencial.

2 Típicamente, los conceptos de corrección y completitud se entienden como conceptos relativos a sistemas lógicos formalizados, pero aquí los utilizamos en un sentido algorítmico: dadas ciertas entradas (inputs), corrección significa que si un algoritmo produce una salida (output), la salida es correcta; y completitud, que un algoritmo garantiza una salida correcta dada una entrada arbitraria.
} 
gramas regionales de Euler, los diagramas triliterales de Carroll, los diagramas lineales de Hamilton, los grafos existenciales de Peirce, los diagramas lineales de Englebretsen, los diagramas lineales de Pagnan y los diagramas de rompecabeza de Castro-Manzano. Adicionalmente, es importante observar que estudiamos estos sistemas de diagramas como sistemas no formalizados.

\subsubsection{Diagramas lineales de Averroes (AVRS)}

i) Fuente: Aristotelis Stagiritae Omnia Quae Extant Opera Nunc primum selectis translationibus, collatisque cum graecis emendatissmis exemplaribus, Marginels scholijs illustrata, \& in nouum ordinem digesta, Additis etiam nonnullis libris nunquam antea latinitate donatis (1552).

ii) Vocabulario: líneas (arcos) y vértices.

iii) Sintaxis: un vértice es un término; una línea que une dos términos es una proposición categórica. Las letras mayúsculas que etiquetan cada vértice representan un término. Las letras mayúsculas que etiquetan un arco representan la cantidad y la cualidad de la proposición: O (Todo es), N (Ningún es), Al (Algún es) y Al.non. (Algún no es).

iv) Silogismos de la primera figura:

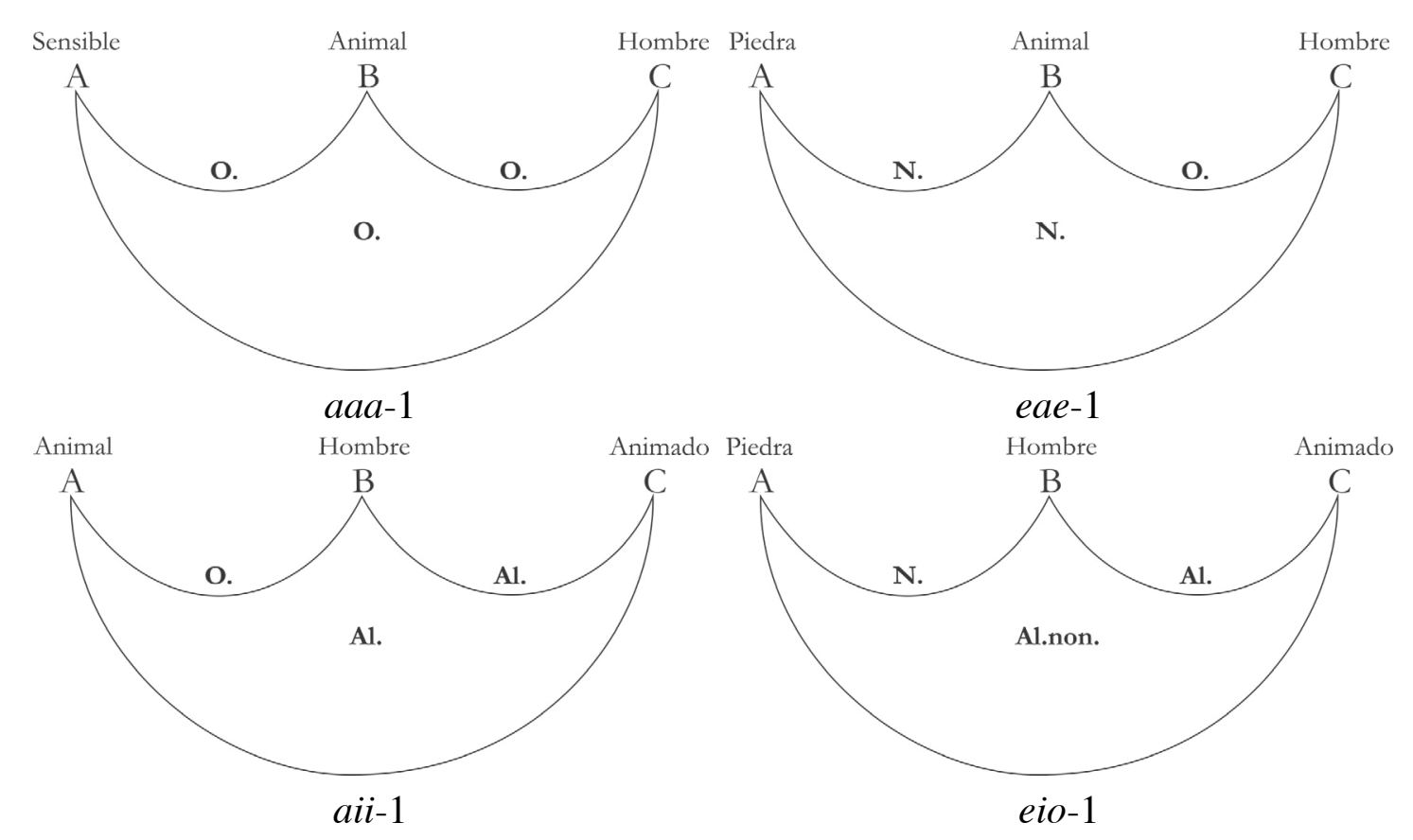

Figura 4. Silogismos de la primera figura en AVRS 
v) Evaluación:

1. AVRS no es correcto. Al codificar los silogismos de la primera figura observamos que si dibujamos las premisas, la conclusión no queda dibujada automáticamente. Consideremos, por ejemplo, el diagrama del modo aaa de la primera figura (aaa-1). Al dibujar la premisa 1 dibujamos un arco del vértice $B$ al vértice $A$ con la etiqueta $O$ (e.d. Todo $B$ es $A$ ), al dibujar la premisa 2 dibujamos un arco del vértice $\mathrm{C}$ al $\mathrm{B}$ con la etiqueta $\mathrm{O}$ (e.d. Todo $C$ es $B$ ), pero al dibujar las premisas no se dibuja el arco de $\mathrm{C}$ a A (e.d. Todo $\mathrm{C}$ es $\mathrm{A}$ ) y, por tanto, nada indica un nexo inferencial entre $\mathrm{C}$ y $\mathrm{A}$.

2. AVRS no es completo. El sistema no tiene free rides. Sin embargo, por mor de caridad, supongamos que existe un free ride de la primera figura, digamos, del silogismo aaa-1. Al dibujar el diagrama de aaa-1 notamos cierta ambigüedad, pues nada en los arcos o los vértices indica con claridad alguna dirección para codificar un silogismo válido.

3. AVRS no es comprensivo. El sistema no puede ser comprensivo porque carece de free rides. Ahora bien, al revisar los supuestos free rides disponibles observamos, además, que estos no se leen de manera directa, pues es necesario acompañarlos de una explicación lingüística adicional que indique, por ejemplo, la dirección de los arcos y que los arcos se tienen que introducir a conveniencia o con conocimiento previo de las reglas de la silogística.

4. AVRS es claro. El sistema es claro en la medida en que cada ítem del vocabulario representa lo que pretende representar en cualquiera de sus ocurrencias.

\subsubsection{Diagramas lineales de Leibniz (LBNZ)}

i) Fuente: De formae logicae comprobatione per linearum ductus (1703?).

ii) Vocabulario: líneas sólidas horizontales y líneas punteadas verticales.

iii) Sintaxis: las líneas horizontales sólidas representan términos y las líneas punteadas verticales representan relaciones entre términos (i.e. proposiciones categóricas). La universal afirmativa (Todo $S$ es $P$ ) se representa colocando una línea horizontal sólida bajo otra línea horizontal sólida de mayor longitud. La universal negativa (Ningún $S$ es $P$ ) se indica colocando una línea horizontal sólida separada de otra línea del mismo tipo. La particular afirmativa, buscando una intersección entre dos líneas (Algún S es P); y la particular negativa, buscando que una parte de una línea no esté dentro de toda la otra línea (Algún $S$ no es $P$ ). iv) Silogismos de la primera figura: 

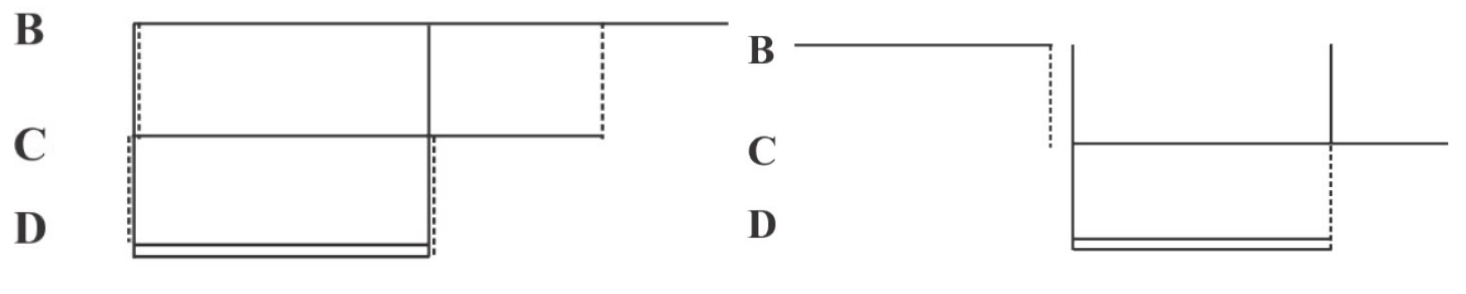

$a a a-1$



$e a e-1$

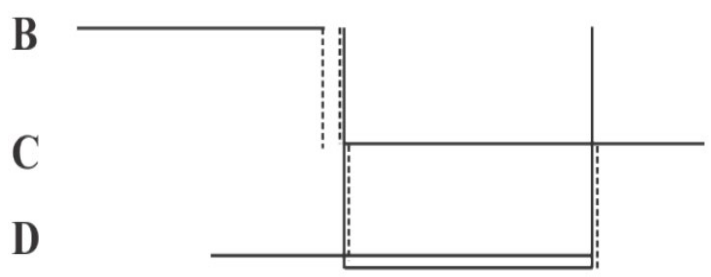

eio-1

Figura 5. Silogismos de la primera figura en LBNZ

v) Evaluación:

1. LBNZ es correcto. Al codificar los silogismos de la primera figura observamos que si dibujamos las premisas, la conclusión queda dibujada automáticamente. Consideremos, a modo de ejemplo, el diagrama del modo aaa-1. Para codificar la premisa 1 dibujamos dos líneas horizontales: una etiquetada con $\mathrm{B}$ y otra paralela, de menor longitud que $\mathrm{B}$, etiquetada con $\mathrm{C}$ (para indicar que Todo C es B); para codificar la premisa 2 dibujamos otra línea paralela a $\mathrm{C}$, y de menor longitud que $\mathrm{C}$, etiquetada con $\mathrm{D}$ (para indicar que Todo $\mathrm{D}$ es $C$ ). Al hacer esto notamos que hemos dibujado un par de líneas, $\mathrm{B}$ y $\mathrm{D}$, de tal modo que la línea $\mathrm{D}$, paralela a y de menor longitud que $\mathrm{B}$, queda dibujada automáticamente bajo $\mathrm{B}$ para indicar que D queda indexada en B (lo que indica que Todo D es B). Y lo mismo ocurre, mutatis mutandis, con el resto de los free rides de la primera figura.

2. LBNZ es completo. Si tomamos los free rides de la primera figura, notamos que codifican silogismos válidos. En efecto, al codificar silogismos válidos obtenemos free rides: por reductio, supongamos que tomamos cualquier silogismo válido pero que uno de ellos no codifica un free ride. Ahora, sabemos que cuando aplicamos el sistema lineal a los silogismos de la primera figura obtenemos free rides, pero como todo silogismo válido puede reducirse a un silogismo de la primera figura, por el Lema de Aristóteles, entonces toda aplicación del sistema lineal a las formas silogísticas válidas debería producir free rides, lo que contradice la suposición inicial. ${ }^{3}$

3 Esto contrasta con el sistema de diagramas regionales de Leibniz, el cual no parece compartir las mismas propiedades lógicas (Castro-Manzano, 2020a). 
3. LBNZ es comprensivo. El sistema es comprensivo porque los free rides disponibles se leen de manera directa y, con cierto entrenamiento, no es necesario acompañarlos de una explicación lingüística adicional, pues la ubicación y la longitud de las líneas ofrecen un mecanismo inferencial que no requiere que un agente razonador tenga conocimiento previo de las reglas de la silogística.

4. LBNZ es claro. El sistema es claro en la medida en que cada ítem del vocabulario representa lo que pretende representar en cualquiera de sus ocurrencias.

\subsubsection{Diagramas lineales de Lambert (LAMB)}

i) Fuente: Neues Organon oder Gedanken Über die Erforschung und Bezeichnung des Wahren und dessen Unterscheidung vom Irrthum und Schein (1704).

ii) Vocabulario: líneas horizontales sólidas y líneas horizontales punteadas.

iii) Sintaxis: las líneas representan términos. Las combinaciones de líneas representan las proposiciones categóricas. Las líneas horizontales sólidas representan términos cuantificados universalmente, mientras que las líneas punteadas representan proposiciones particulares. Adicionalmente, la cualidad se representa por la posición de las líneas: dado un par de líneas, si una línea aparece por debajo de otra, la primera está incluida en la segunda (Todo $S$ es $P$, si ambas líneas son sólidas, Algún $S$ es $P$ si una de ellas es punteada); de otro modo, no están relacionadas (Ningún S es $P$, si ambas líneas son sólidas, Algún $S$ no es $P$ si una de ellas es punteada).

iv) Silogismos de la primera figura:
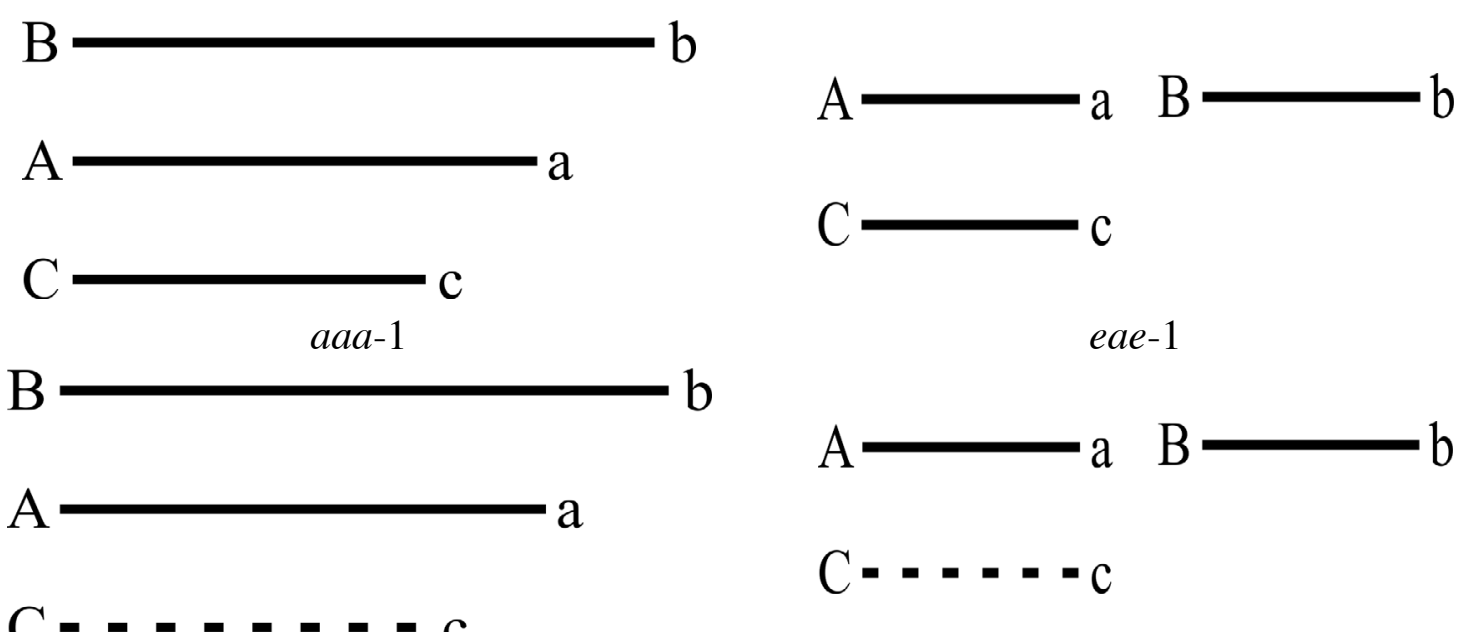

aii-1

eio-1

Figura 6. Silogismos de la primera figura en $L A M B$ 
v) Evaluación:

1. LAMB es correcto. Al codificar los silogismos de la primera figura observamos que si dibujamos las premisas, la conclusión queda dibujada automáticamente. Consideremos el diagrama de un silogismo tipo aaa-1. Al dibujar la primera premisa encontramos que la línea $B$ es mayor que la línea $A$ (e.d. Todo $A$ es $B$ ), posteriormente dibujamos otra línea por debajo de la anterior que indique el término C (e.d. Todo C es A). Por tanto, automáticamente, queda dibujada una proposición de tipo universal afirmativa que indica el nexo inferencial entre B y C (e.d. Todo Ces B).

2. LAMB no es completo. Tomemos una forma inválida, digamos eee-1. Al dibujar la primera premisa dibujamos dos líneas para los términos C y A (e.d. Ningún $C$ es $A$ ); al dibujar la segunda premisa se dibuja una tercera línea para el tercer término B (e.d. Ningún A es B) y, al hacer esto, queda automáticamente dibujada una proposición de tipo universal negativa (e.d. Ningún $C$ es $B$ ) (Fig. 7). Sin embargo, esta forma es inválida en nuestra base deductiva.

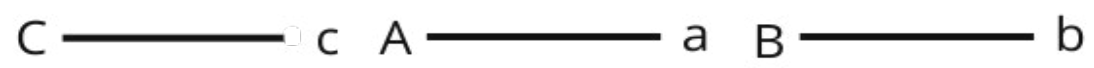

Figura 7. eee-1 en LAMB

1. LAMB es comprensivo. Los free rides que LAMB ofrece se pueden interpretar de manera directa, lo cual garantiza que no haya necesidad de aportar explicaciones lingüísticas adicionales.

2. LAMB es claro. El sistema es claro en la medida en que cada ítem del vocabulario representa lo que pretende representar en cualquiera de sus ocurrencias.

\subsubsection{Diagramas regionales de Euler (EULR)}

i) Fuente: Briefe an eine deutsche Prinzessin (1768).

ii) Vocabulario: líneas cerradas (circunferencias o discos).

iii) Sintaxis: las circunferencias representan términos. Las relaciones entre circunferencias representan proposiciones categóricas. La cantidad y la cualidad se representan por la posición de las circunferencias: dado un par de circunferencias, si una circunferencia está completamente dentro de otra, la primera está incluida en la segunda (Todo $S$ es $P$ ); de otro modo, no están relacionadas (Ningún $S$ es $P$ ). Y si una circunferencia aparece parcialmente dentro de otra, presentan una intersección (Algún $S($ no ) es $P$ ). 
iv) Silogismos de la primera figura:

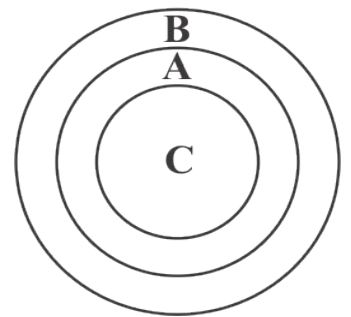

$a a a-1$

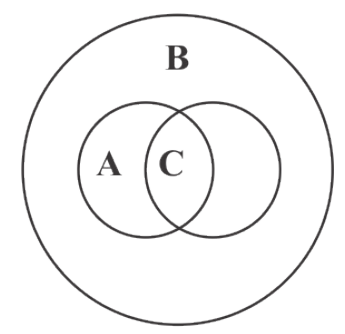

aii-1

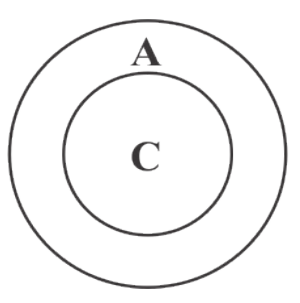

$e a e-1$

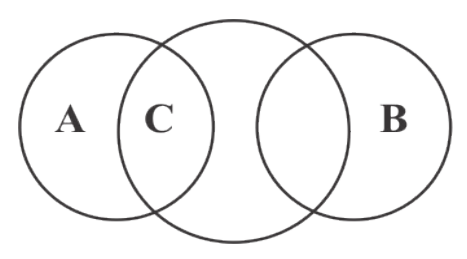

eio-1

Figura 11. Silogismos de la primera figura en EULR

v) Evaluación:

1. EULR no es correcto. No es correcto porque ciertos free rides no son silogismos válidos: tomemos, por ejemplo, un diagrama con tres circunferencias B, C y D tales que C está dentro de B (para indicar que Todo C es B) y D está separada de C (y B) (para indicar que Ningún D es C); luego, obtenemos un diagrama en el que las circunferencias B y D están separadas para indicar que Ningún $B$ es $D$, pero tal estructura argumental no corresponde con un silogismo válido en nuestra base deductiva.

2. EULR no es completo. Al codificar ciertos silogismos válidos de la primera figura observamos que, si dibujamos las premisas, la conclusión deseada no queda dibujada automáticamente. Consideremos, a modo de contra-ejemplo, el diagrama del modo eio-1. Para codificar la premisa 1 dibujamos dos circunferencias: una etiquetada con $\mathrm{C}$ y otra etiquetada con B (para indicar que Ningún $C$ es $B$ ); para codificar la premisa 2 dibujamos otra circunferencia etiquetada con $\mathrm{D}$ haciendo un traslape con la circunferencia $\mathrm{C}$ (para indicar que Algún D es $C$ ). Al hacer esto notamos que las circunferencias D y $B$ quedan separadas automáticamente, pero esto no indica la conclusión deseada (que Algún D no es B), antes bien, representa la conclusión Ningún D es $B$, lo cual no debería ocurrir, pues tal conclusión corresponde a un modo eie, que no es válido en la base deductiva. 
3. EULR es comprensivo. Aunque este sistema no ofrece un mecanismo inferencial confiable para modelar la inferencia silogística, los free rides disponibles en el sistema se leen de manera directa y sin necesidad de apelar a consideraciones lingüísticas adicionales.

4. EULR es claro. El sistema es claro en la medida en que cada ítem del vocabulario representa lo que pretende representar en cualquiera de sus ocurrencias.

\subsubsection{Diagramas lineales de Hamilton (HMTN)}

i) Fuente: Lectures on Metaphysics and Logic (1860).

ii) Vocabulario: Coma, dos puntos y líneas horizontales triángulares (flechas).

iii) Sintaxis: las literales representan términos, las flechas indican la posición del término sujeto (en la base) y la posición del término predicado (en la punta). Los signos de puntuación indican la cantidad de la proposición: las comas indican la cantidad particular, mientras que los dos puntos, “:”, indican la cantidad universal. Finalmente, la cualidad negativa se indica por una línea perpendicular a las flechas; de otro modo, si no hay tal línea perpendicular, la cualidad de la proposición es afirmativa. Así, S: $\bullet, P$ es Todo $S$ es $P, \mathrm{~S}: \nabla \mid, P$ es Ningún $S$ es $P$, S,॰,P es Algún $S$ es $P$ y $\mathrm{S}, \bullet \mid, \mathrm{P}$ es Algún $S$ no es $P$.

iv) Silogismos de la primera figura:
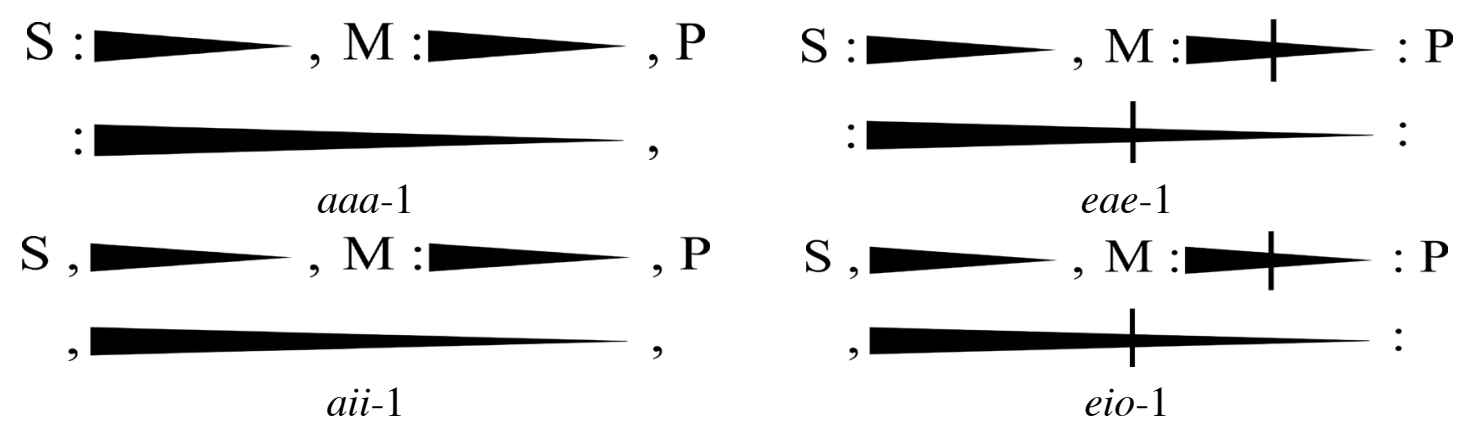

Figura 8. Silogismos de la primera figura en HMTN

v) Evaluación:

1. HMTN no es correcto. Si bien al codificar los silogismos de la primera figura queda automáticamente dibujada una relación diagramática que representa la conclusión, no sucede lo mismo con las figuras posteriores, esto se debe a que los free rides no son lo suficientemente claros con respecto a la direccionalidad de las flechas. Supongamos, por ejemplo, que tenemos un silogismo de la forma eio-2. En este ejemplo notamos cómo la direccionalidad se comporta de manera extraña en las premisas, lo que dificulta la interpretación de la conclusión (Fig. 9). 


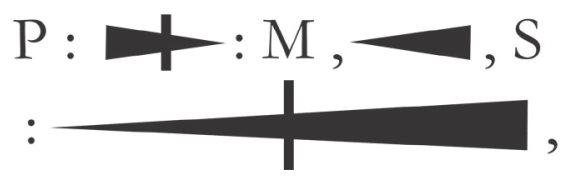

Figura 9. eio-2 en HMTN

2. HMTN no es completo. Consideremos una forma silogística inválida, digamos iii-1. Lo primero que se debe hacer es codificar la premisa 1 (e.d. Algún $M$ es $P$ ), representando ambos términos con comas. Después aplicamos el mismo procedimiento para representar la premisa 2 (e.d. Algún $S$ es $M$ ). Ahora, al recorrer los signos externos y dibujar la conclusión, obtenemos una proposición particular de nuevo (e.d. Algún S es $\mathrm{P}$ ), lo cual es incorrecto en nuestra base deductiva (Fig. 10).

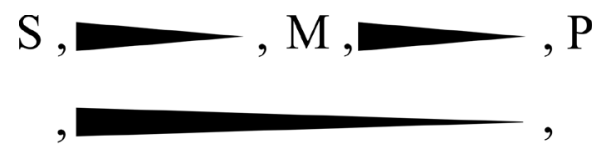

Figura 10. iii-1 en HMTN

3. HMTN no es comprensivo. Al revisar los supuestos free rides podemos concluir que se pueden hacer inferencias de manera directa, sin necesidad de tener explicaciones lingüísticas adicionales. Sin embargo, existe un problema en la interpretación de las flechas, ya que el sistema no ofrece una regla que indique con claridad el funcionamiento de su direccionalidad.

4. HMTN es claro. Cada objeto diagramático representa lo que pretende representar.

\subsubsection{Diagramas triliterales de Carroll (CRLL)}

i) Fuente: The Game of Logic (1887).

ii) Vocabulario: tablero (diagrama triliteral) y fichas.

iii) Sintaxis: cada celda del tablero representa una posición de un término mientras que las fichas indican si tales celdas están vacías o llenas. El tablero es una matriz de dos entradas $\mathrm{S}$ y su complemento, S; y P y su complemento, P. Las fichas negras indican que una celda está llena; las grises, que una celda está vacía. Así, una proposición universal afirmativa, Todo $S$ es $P$, se representa colocando una ficha negra en la celda que es $\mathrm{S}$ y $\mathrm{P}$ y una ficha gris en la que es $S$ y no es P. La universal negativa, Ningún $S$ es $P$, se representa con una ficha gris en la celda que es $\mathrm{S}$ y $\mathrm{P}$ y una ficha negra en la celda que es $\mathrm{S}$ y no es $\mathrm{P}$. La particular afirmativa, Algún S es 
$P$, se representa colocando una ficha negra en la celda que es $\mathrm{S}$ y $\mathrm{P}$; y por último, la particular negativa, Algún $S$ no es $P$, acomodando una ficha negra en la celda que es $S$ y no es $P$.

iv) Silogismos de la primera figura:

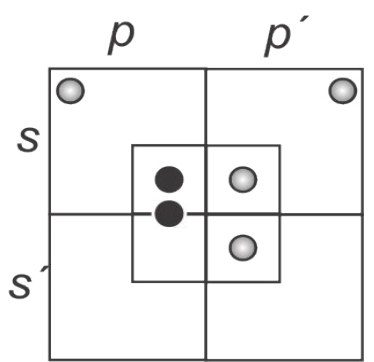

$a a a-1$

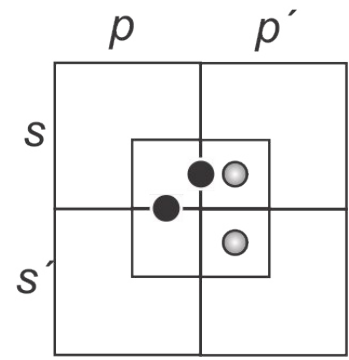

aii-1

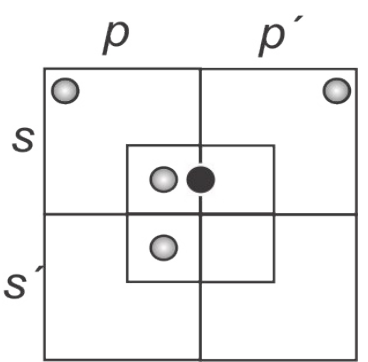

$e a e-1$

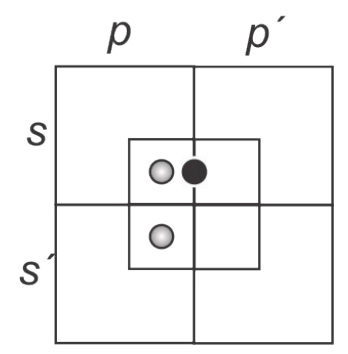

eio-1

Figura 11. Silogismos de la primera figura en CRLL

v) Evaluación:

1. CRLL es correcto. Al codificar los silogismos de la primera figura observamos que si dibujamos las premisas, la conclusión queda dibujada automáticamente. Consideremos, a modo de ejemplo, el diagrama del modo aaa-1. Para construirlo, dibujamos (i.e. acomodamos) la siguiente serie de diagramas (y fichas):
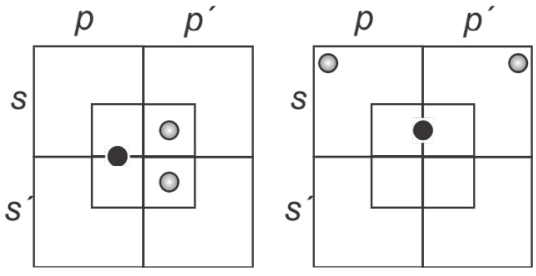

Todo $M$ es $P$
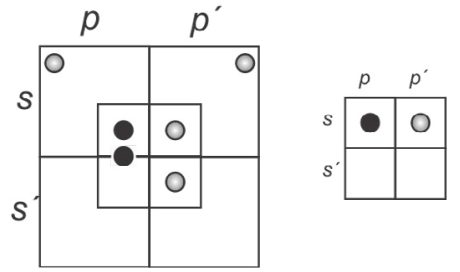

Todo S es

Figura 12. aaa-1 en CRLL

De izquierda a derecha, comenzamos dibujando los diagramas para las proposiciones Todo $M$ es $P$ y Todo $S$ es $M$; posteriormente dibujamos la combinación de ambos diagramas y 
notamos que automáticamente quedan dibujadas un par de fichas, una negra y una gris, que indican que aquello que es $\mathrm{S}$ también es $\mathrm{P}$ y que no hay $\mathrm{S}$ que no sea $\mathrm{P}$ (lo que indica que Todo S es $P$ ). Lo mismo ocurre, mutatis mutandis, con el resto de los free rides de la primera figura.

2. CRLL es completo. Si tomamos los free rides que corresponderían a la primera figura, notamos que codifican silogismos válidos.

3. CRLL es comprensivo. Los free rides que CRLL ofrece se pueden interpretar de manera directa, por lo que no hay necesidad de aportar explicaciones lingüísticas adicionales.

4. CRLL es claro. El sistema es claro en la medida en que cada ítem del vocabulario representa lo que pretende representar en cualquiera de sus ocurrencias.

\subsubsection{Grafos existenciales de Peirce (PEIR)}

i) Fuente: Prolegomena to an Apology for Pragmaticism (1906).

ii) Vocabulario: el plano, líneas cerradas y líneas abiertas.

iii) Sintaxis: los grafos existenciales de Peirce vienen en una variedad de formas: Alpha es el sistema básico, mientras que Beta y Gamma son extensiones del primero. Esto tiene sentido porque Alpha tiene que ver con la lógica proposicional, Beta con la lógica de primer orden y Gamma trata con la lógica modal. En Alpha, y por lo tanto en el resto de sistemas, hay tres elementos básicos: la hoja de afirmación, el corte y las literales. La hoja de afirmación es el plano en el que ocurren las dos características básicas de la lógica: el rechazo (representado por una línea cerrada, llamada corte, dentro de una hoja de afirmación), y la afirmación (representada por las literales dentro de una hoja de afirmación).

iv) Silogismos de la primera figura: aunque no mencionaremos las reglas $R$ o permisiones que nos permiten producir conclusiones (cfr. Roberts, 1973), porque no es necesario para nuestros fines, a continuación mostramos las siguientes derivaciones solo por mor de ejemplificación: 


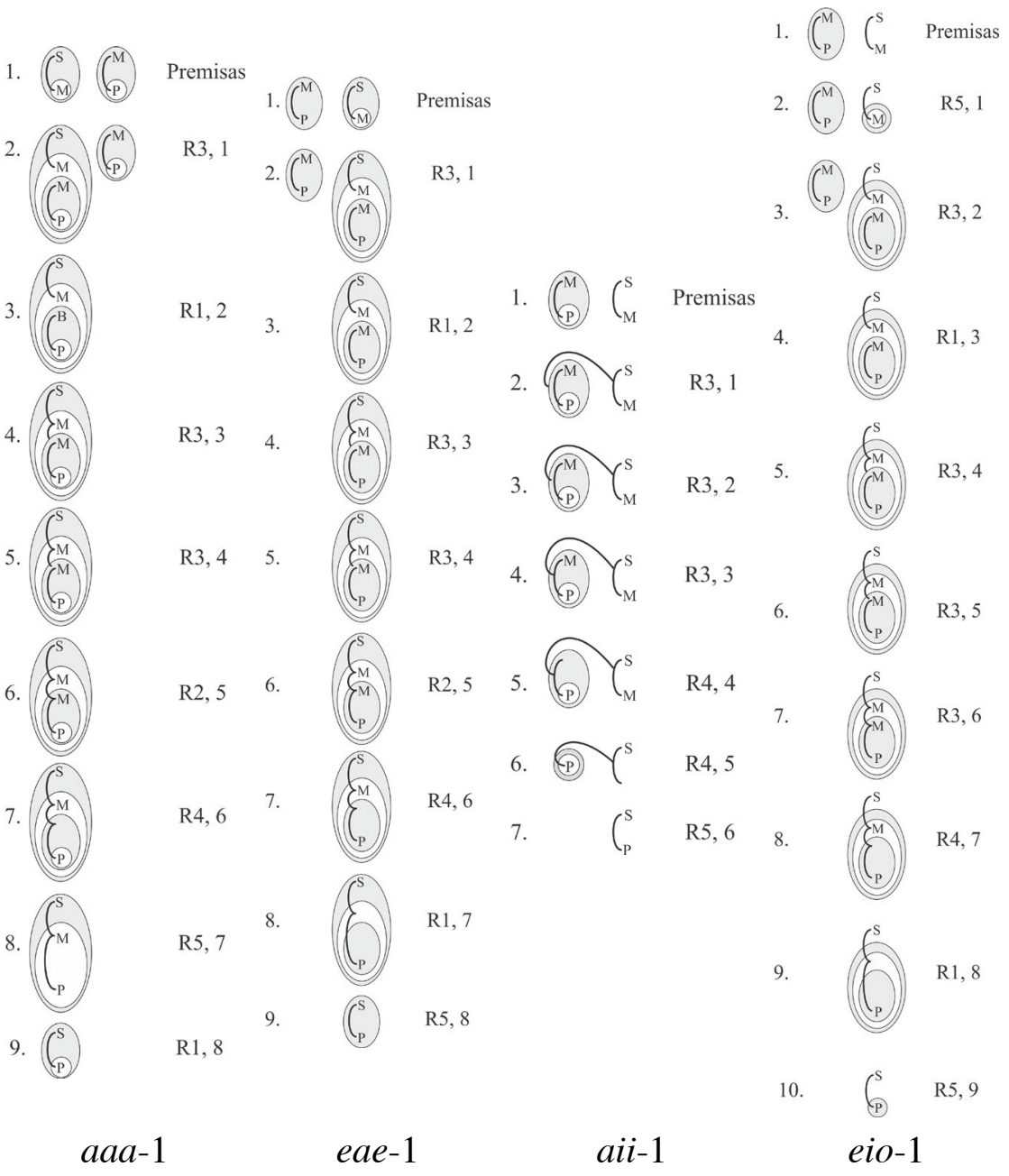

Figura 13. Silogismos de la primera figura en PEIR

v) Evaluación:

1. PEIR no es correcto. Este sistema no es correcto porque los free rides no codifican silogismos válidos; sin embargo, en este punto es preciso comentar que aunque los grafos existenciales no son diagramas lógicos en el sentido que estamos usando en este trabajo, sí definen una lógica en otro sentido, como en una álgebra diagramática o algegraph, como hemos argumentado en otro lugar (Castro-Manzano, 2020b). En efecto, la diagramación de premisas no indica nada acerca de cuál debería ser la conclusión correcta. Al contrario, para alcanzar un diagrama adecuado de una conclusión debemos transformar los diagramas iniciales, paso a paso, de manera algebraica.

2. PEIR no es completo. Por lo dicho anteriormente, al codificar ciertos silogismos válidos de la primera figura observamos que, si dibujamos las premisas, la conclusión deseada no queda dibujada automáticamente. Volveremos a este punto en las conclusiones. 
3. PEIR es comprensivo. Aunque este sistema no ofrece free rides de manera directa y sin necesidad de apelar a consideraciones lingüísticas adicionales, es comprensivo en el sentido algebraico.

4. PEIR es claro. El sistema es claro en la medida en que cada ítem del vocabulario representa lo que pretende representar en cualquiera de sus ocurrencias.

\subsubsection{Diagramas lineales de Englebretsen (ENGL)}

i) Fuente: Line Diagrams for Logic: Drawing Conclusions (1982).

ii) Vocabulario: líneas y puntos.

iii) Sintaxis: sean $S(\bar{S})$ y $P(\bar{P})$ términos. Los puntos representan términos. La estructura $-\mathrm{S}-\mathrm{P}(-\mathrm{S}-\overline{\mathrm{P}})$ representa una proposición universal afirmativa (negativa) y la estructura $-\backslash \mathrm{S}-\mathrm{P}(-\backslash \mathrm{S}-\overline{\mathrm{P}})$ representa una proposición particular afirmativa (negativa).

iv) Silogismos de la primera figura:

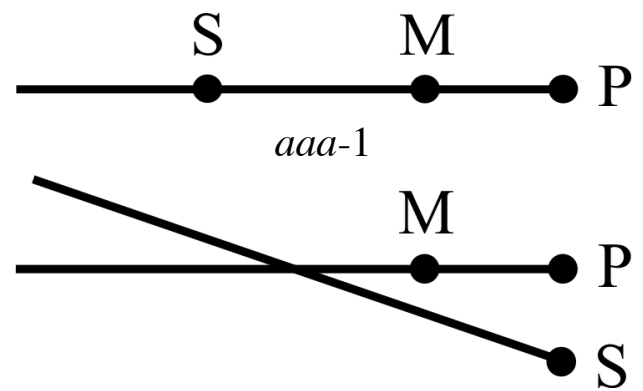

aii-1

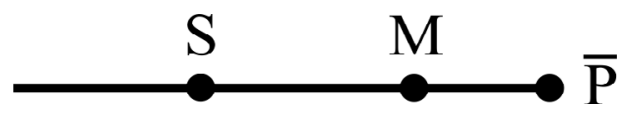

eae-1

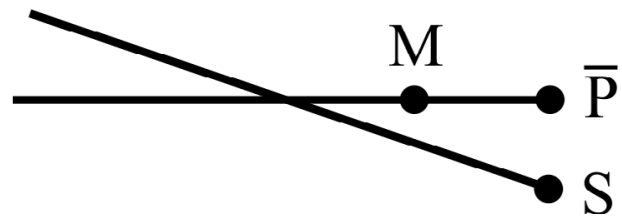

eio-1

Figura 14. Silogismos de la primera figura en ENGL

v) Evaluación:

1. ENGL es correcto. Al codificar los silogismos de la primera figura observamos que si dibujamos las premisas, la conclusión queda dibujada automáticamente. Consideremos, a modo de ejemplo, el diagrama del modo aaa-1. Para codificar la premisa 1 dibujamos una línea horizontal con un punto a la mitad de la línea, $\mathrm{M}$, y un punto al final, $\mathrm{P}$, para indicar que Todo $M$ es $P$ ); para codificar la premisa 2 dibujamos un punto sobre la misma línea, $S$, pero antes del punto M para indicar que Todo $S$ es $M$. Al hacer esto notamos que hemos dibujado el punto $S$ antes del punto $\mathrm{P}$ sobre la misma línea horizontal, lo que indica que Todo $S$ es $P$. Y lo mismo ocurre, mutatis mutandis, con el resto de los free rides de la primera figura.

2. ENGL es completo. Por un razonamiento similar al de LBNZ, si tomamos los free rides de la primera figura, notamos que codifican silogismos válidos. 
3. ENGL es comprensivo. Los free rides del sistema se leen de manera directa y sin necesidad de apelar a consideraciones lingüísticas adicionales.

4. PEIR es claro. El sistema es claro en la medida en que cada ítem del vocabulario representa lo que pretende representar en cualquiera de sus ocurrencias.

\subsubsection{Diagramas lineales de Pagnan (PAGN)}

i) Fuente: A Diagrammatic Calculus of Syllogisms (2012).

ii) Vocabulario: flechas y puntos.

iii) Sintaxis: sea $T$ un término. $T \rightarrow T$ representa una proposición universal afirmativa; $T \rightarrow . \leftarrow T$, la universal negativa; $\mathrm{T} \leftarrow . \rightarrow \mathrm{T}$, la particular afirmativa; $\mathrm{y} \leftarrow . \rightarrow . \leftarrow \mathrm{T}$, la particular negativa. iv) Silogismos de la primera figura:

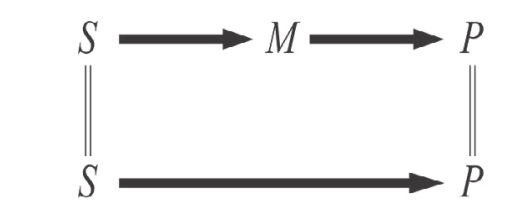

aaa-1

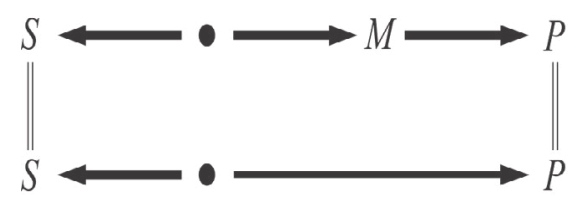

aii-1



eae-1

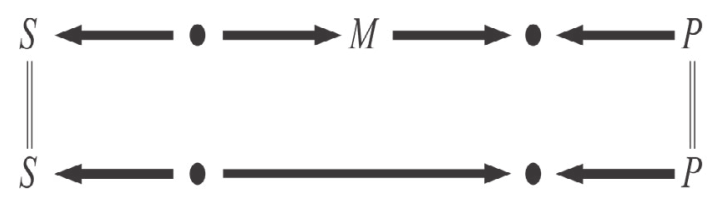

eio-1

Figura 15. Silogismos de la primera figura en PAGN

v) Evaluación:

1. PAGN es correcto. Al codificar los silogismos de la primera figura observamos que si dibujamos las premisas, la conclusión queda dibujada automáticamente. Consideremos, a modo de ejemplo, el diagrama del modo aaa-1. Para codificar la premisa 1 dibujamos una flecha del término $S$ al término $\mathrm{M}$ (para indicar que Todo $S$ es $M$ ) y una flecha del término $\mathrm{M}$ al término $\mathrm{P}$ (para indicar que Todo $M$ es $P$ ). Con esto podemos ver que hemos dibujado una flecha que va de $\mathrm{S}$ a $\mathrm{M}$, lo que indica que Todo $S$ es $P$. Lo mismo ocurre, mutatis mutandis, con el resto de los free rides de la primera figura.

2. PAGN es completo. Por un razonamiento similar al de LBNZ (y ENGL).

3. PAGN es comprensivo. Los free rides del sistema se leen de manera directa y sin necesidad de apelar a consideraciones lingüísticas adicionales.

4. PAGN es claro. El sistema es claro en la medida en que cada ítem del vocabulario representa lo que pretende representar en cualquiera de sus ocurrencias. 


\subsubsection{Diagramas de rompecabeza (JGSW)}

i) Fuente: Logic with Jigsaw Puzzles (2018)

ii) Vocabulario: piezas de rompecabezas: sockets $(\sqsubset, \sqsupset)$ y knobs (-).

iii) Sintaxis: cada pieza representa un término. ᄃ- representa la proposición universal afirmativa; $\sqsubset \sqsupset$, la universal negativa; --, la particular afirmativa; $\mathrm{y}-\sqsupset$, la particular negativa. iv) Silogismos de la primera figura:

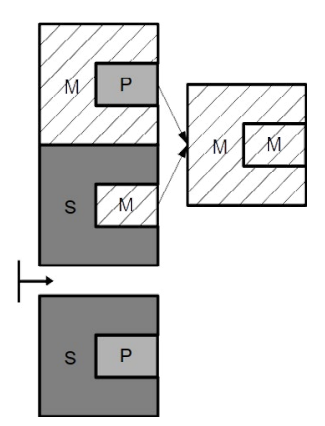

$a a a-1$

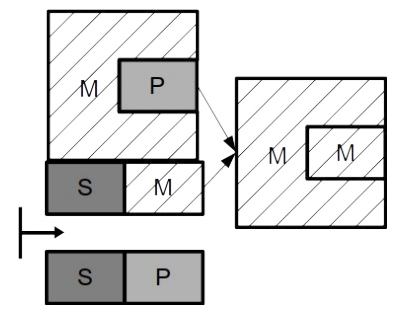

aii-1

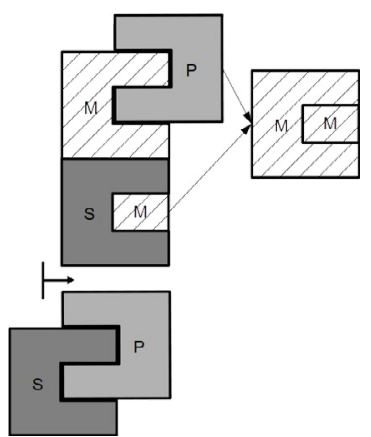

eae-1

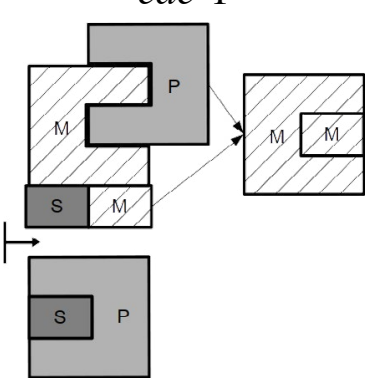

eio-1

v) Evaluación:

1. JGSW es correcto. Al codificar los silogismos de la primera figura observamos que si dibujamos las premisas, la conclusión queda dibujada automáticamente si al ensamblar las piezas de los términos medios, $\mathrm{M}$, estas forman una estructura ᄃ $ᄃ$-. Consideremos, a modo de ejemplo, el diagrama del modo aaa-1. Para codificar la premisa 1 dibujamos $ᄃ_{\mathrm{M}-\mathrm{P}}$ (para indicar que Todo $M$ es $P$ ) y $\subset_{\text {s-M }}$ (para indicar que Todo $S$ es $M$ ). Al ensamblar los términos podemos ver que hemos dibujado $\Sigma_{\mathrm{M}-\mathrm{M}}$, lo que indica que el resto del diagrama ha producido $ᄃ_{\text {S-p }}$ es decir, que Todo $S$ es $P$. Lo mismo ocurre, mutatis mutandis, con el resto de los free rides de la primera figura.

2. JGSW es completo. Por un razonamiento similar al de LBNZ (y PAGN y ENGL).

3. JGSW es comprensivo. Los free rides del sistema se leen de manera directa y sin necesidad de apelar a consideraciones lingüísticas adicionales; sin embargo, como ocurre con el sistema CRLL, es necesario construir las configu- 
raciones. Esta observación es interesante porque sugiere que JGSW y CRLL no son sistemas lineales ni regionales, sino diagramas, digamos, cinestésicos, ya que no basta con dibujar los esquemas visuales: es preciso trabajar con ellos de manera manual.

4. JGSW es claro. El sistema es claro en la medida en que cada ítem del vocabulario representa lo que pretende representar en cualquiera de sus ocurrencias.

\section{Conclusiones}

A modo de resumen, consideremos los siguientes cuadros.

\begin{tabular}{|c|c|c|c|c|c|c|}
\hline \multirow{2}{*}{ TIPO } & \multirow{2}{*}{ SISTEMA } & \multirow{2}{*}{ CCA. } & \multicolumn{2}{|c|}{ PROPIEDADES LÓGICAS } & \multicolumn{2}{|c|}{ PROPIEDADES REPRESENTATIVAS } \\
\hline & & & CORRECCIÓN & COMPLETITUD & COMPRENSIÓN & CLARIDAD \\
\hline \multirow{6}{*}{ Lineal } & AVRS & 1552 & - & - & - & $x$ \\
\hline & LBNZ & 1703 & $x$ & $x$ & $x$ & $x$ \\
\hline & LMBT & 1704 & $x$ & - & $x$ & $x$ \\
\hline & HMNT & 1858 & - & - & - & $x$ \\
\hline & ENGL & 1982 & $x$ & $x$ & $x$ & $x$ \\
\hline & PAGN & 2012 & $x$ & $x$ & $x$ & $x$ \\
\hline \multirow{2}{*}{ Regional } & EULR & 1768 & - & - & $x$ & $x$ \\
\hline & PEIR & 1906 & - & - & $x$ & $x$ \\
\hline \multirow{2}{*}{ Cinestésico } & CRLL & 1887 & $x$ & $x$ & $x$ & $x$ \\
\hline & JGSW & 2018 & $x$ & $x$ & X & $x$ \\
\hline
\end{tabular}

Cuadro 2. Partición por tipo de diagrama

\begin{tabular}{|c|c|c|c|c|c|c|}
\hline \multirow{2}{*}{ CCA. } & \multirow{2}{*}{ SISTEMA } & \multirow{2}{*}{ TIPO } & \multicolumn{2}{|c|}{ PROPIEDADES LÓGICAS } & \multicolumn{2}{c|}{ PROPIEDADES REPRESENTATIVAS } \\
\cline { 4 - 7 } & & & CORRECCIÓN & COMPLETITUD & COMPRENSIÓN & CLARIDAD \\
\hline 1552 & AVRS & Lineal & - & - & - & $X$ \\
\hline 1703 & LBNZ & Lineal & $X$ & $X$ & $X$ & $X$ \\
\hline 1704 & LMBT & Lineal & $X$ & - & $X$ & $X$ \\
\hline 1768 & EULR & Regional & - & - & $X$ & $X$ \\
\hline 1858 & HMNT & Lineal & - & - & $X$ & $X$ \\
\hline 1887 & CRLL & Cinestésico & $X$ & $X$ & $X$ & $X$ \\
\hline 1906 & PEIR & Regional & - & - & $X$ & $X$ \\
\hline 1982 & ENGL & Lineal & $X$ & $X$ & $X$ & $X$ \\
\hline 2012 & PAGN & Lineal & $X$ & $X$ & $X$ & $X$ \\
\hline 2018 & JGSW & Cinestésico & $X$ & $X$ & $X$ & $X$ \\
\hline
\end{tabular}

Cuadro 3. Partición de sistemas por el tiempo 
A partir de este resumen, consideremos las siguientes anotaciones finales:

i) Ser diagramático se dice de varias maneras. En la sección 2 ya habíamos argumentado que no todos los diagramas son de la misma naturaleza debido a que no todos comparten las mismas funciones. Pero prestando más atención a los detalles de este estudio comparativo podemos notar que ni siquiera todos los diagramas que se precian de tener una misma función, en este caso inferencial, tienen las mismas propiedades. En consecuencia, podemos hacer una clasificación de los diagramas más fina, no solo en términos de sus funciones, sino en términos de sus propiedades, tanto inferenciales como representativas.

ii) Las propiedades lógicas implican a las representativas. Al hacer un balance de estas propiedades podemos observar que las lógicas implican a las representativas, aunque no necesariamente al revés (Cuadros 2 y 3 ). Parece que podemos tener sistemas claros y comprensivos que no sean correctos ni completos; pero parece que no podemos tener sistemas correctos y completos que no sean claros y comprensivos. Luego, no todos los diagramas inferenciales son equivalentes con respecto a lo que se puede probar con ellos. Esto es interesante porque sugiere que la definición inicial de Gardner contiene una cláusula necesaria (a saber, que un diagrama es una figura bidimensional con cierto isomorfismo con las proposiciones) pero no suficiente para definir un diagrama lógico. Volveremos a este punto en la anotación $i v$ ).

iii) El poder inferencial y representativo de un diagrama es sensible al objeto diagramático. Así, dados los comentarios anteriores, podemos notar una jerarquía entre los diagramas: los sistemas lineales y cinestésicos suelen tener más propiedades lógicas que los regionales aunque, en números totales, los regionales son más comprensivos que los lineales. Una posible explicación de esta situación tiene que ver con los objetos diagramáticos que cada sistema utiliza: los sistemas lineales hacen uso de líneas abiertas; los regionales, de líneas cerradas; y los cinestésicos hacen uso de una combinación de objetos.

iv) El poder inferencial y representativo de un diagrama es sensible a sus reglas. Como comentamos previamente, el sistema de Peirce parece que está en otra categoría. Notemos que los grafos existenciales no son inferenciales en el sentido que estamos asumiendo en esta contribución pues, como hemos mencionado previamente, la acción de dibujar las premisas no produce la conclusión correcta; sin embargo, aquí es preciso observar una similitud 
que tiene la noción de free ride con la noción de validez modelo-teorética: del mismo modo en que decimos que una inferencia es válida si y solo si todo modelo de las premisas es modelo de la conclusión, una inferencia diagramática es un free ride si y solo si todo dibujo de las premisas es un dibujo de la conclusión. Por tanto, una de las razones por las cuales los diagramas de Peirce no son lógicos tiene que ver más con una noción de validez que con un defecto o un problema de los diagramas mismos. Tal parece que los grafos de Peirce se entienden mejor en términos de una noción de validez prueba-teorética en la medida en que su estructura inferencial tiene un comportamiento algebraico, mientras que el resto de diagramas que hemos revisado aquí se entienden mejor en términos de una noción de validez modelo-teorética (Castro-Manzano, 2020b). En otras palabras, los diagramas de Peirce definen un álgebra de diagramas, más que un sistema diagramático típico. Uno podría argumentar, por tanto, que no deberíamos considerarlo en este estudio comparativo; sin embargo, si no lo hubiésemos incluido, posiblemente no habríamos llegado a estas distinciones.

v) Ser un diagrama lógico no es trivial. Pues bien, combinando estas anotaciones podemos ofrecer una definición de diagrama lógico: un diagrama lógico es un diagrama (en el sentido de Gardner) dentro de un sistema diagramático que es correcto y completo con respecto a una clase de inferencias válidas dada a una base deductiva. Esta definición parece ser adecuada porque nos permite incluir a aquellos sistemas de diagramas que, por sus propiedades, típicamente consideramos como diagramas lógicos bona fide (como los diagramas de Venn-Euler-Peirce (Shin, 1994; Hammer, 1995), por ejemplo) pero excluye a los diagramas que no solo son incorrectos o incompletos, sino aquellos que ni siquiera son inferenciales.

Así pues, para concluir, estas anotaciones nos permiten ofrecer una respuesta a la pregunta de qué es lo que hace que un diagrama sea un diagrama lógico y nos ofrece razones para justificar o considerar a los diagramas lógicos como portadores legítimos de inferencia. Por supuesto, aunque nuestra definición de diagrama lógico ha surgido del estudio comparativo de sistemas concretos y hemos dejado de lado otros sistemas de diagramas (como los diagramas de Maass (1836), De Morgan (1860), Marquand (1881), Macfarlane (1890), Newlin (1906), Hocking (1909), Gonseth (1937), Karnaugh (1954), Savio (1998), entre otros), pensamos que la inclusión de otros sistemas no producirá gran variación en el resultado. $Y$ además, creemos que dicha inclusión podría fomentar el interés en el estudio de los diagramas lógicos, no solo en términos históricos, sino también en términos formales. 
100 - Cogency, Journal of reasoning and argumentation

\section{Referencias}

Allwein, G. y Barwise, J. Logical Reasoning with Diagrams. Nueva York: Oxford University Press, 1996.

Castro-Manzano, J.M. “Syllogistic with Jigsaw Puzzle Diagrams." En Chapman P. et al (eds.) Diagrammatic Representation and Inference. Diagrams 2018. Springer, 2018.

Castro-Manzano, J.M. "Los diagramas lógicos de Leibniz: una comparación." En Casales García , R., Reyes Cárdenas, P. y Velasco, L.A. (eds.) La actualidad de Leibniz. Alcances y perspectivas sobre la obra filosófica y científica de Leibniz. España: Comares, 2020a.

Castro-Manzano, J.M. "On Why Alpha Graphs are not Logic Diagrams." En Reyes-Cárdenas, P. y Herbert, D. (eds.) The Reception of Peirce and Pragmatism in Latin-America. A Trilingual Collection. México: Editorial Torres y Asociados, $2020 \mathrm{~b}$.

Chandrasekaran, B., Janice Glasgow y N. H. Narayanan. Diagrammatic Reasoning: Cognitive and Computational Perspectives. Cambridge: AAAI Press/MIT Press, 1995.

Englebretsen, G. "Linear Diagrams for Syllogisms (with Relationals)." Notre Dame J. Formal Logic 33(1) (1992): 37-69.

Khemlani, S. y Johnson-Laird, P. N. “Theories of the Syllogism: A Meta-Analysis.” Psychological Bulletin 138(3) (2012): 427- 457.

Lagrange, J. L., Boissonnade, A. C. y Vagliente, V. N. Analytical mechanics. Dordrecht: Kluwer Academic, 1997.

Carroll, L. The Game of Logic. Londres: Macmillan \& Co., 1887.

De Morgan, A. Syllabus of a Proposed System of Logic. Londres: Walton \& Maberly, 1860.

Descartes, R., Miller, V. R. y Miller, R. P. Principles of Philosophy. Dordrecht: Reidel, 1983.

Dieudonné, J. Foundations of Modern Analysis. Nueva York: Academic Press, 1996.

Gardner, M. Mathematics Magic and Mistery. Nueva York: Dover, 1956.

Gardner, M. Logic Machines and Diagrams. Nueva York: McGraw-Hill, 1958.

Gonseth, F. Quest-ce Que La Logique. Paris: Harmant, 1937. 
Hammer, E. “Diagrammatic Logic.” En D. M. Gabbay y F. Guenthner (eds.) Handbook of Philosophical Logic (Vol. 4). Amsterdam: Elsevier North Holland, 1995.

Hamilton, W., Mansel, H. L. y Veitch, J. Lectures on Metaphysics and Logic. Boston: Gould \& Lincoln, 1860.

Hammack, R. H. Book of Proof. Richmond: Virginia Commonwealth University, 2013.

Hocking, W. E. “Two Extensions of the Use of Graphs in Elementary Logic." University of California Publications in Philosophy 2 (2) (1909): 31-44.

Kant, I. y Young, J. M. Lectures on Logic. Cambridge: Cambridge University Press, 1992.

Karnaugh, M. “Map method for synthesis of logic circuits.” Electrical Engineering 73(2) (1954): 136-136.

Kepler, J., Aiton, E., Duncan, A. y Field, J. The Harmony of the World. American Philosophical Society, 1997.

Lambert, J. H. Neues Organon oder Gedanken Über die Erforschung und Bezeichnung des Wahren und dessen Unterscheidung vom Irrthum und Schein. Leipzig: Bey Johann Wendler, 1764.

Larkin, J. H. y Simon, H. A. "Why a Diagram is (Sometimes) Worth Ten Thousand Words." Cognitive Science 11(1) (1987): 65-100.

Leibniz, G. W., Remnant, P. y Bennett, J. New Essays on Human Understanding. Cambridge: Cambridge University Press, 1981.

Llull, R. Ars magna. Barchne: Petru Posa, 1501.

Londey, D. y Johanson, C. The Logic of Apuleius: Including a Complete Latin Text and English Translation of the Peri hermeneias of Apuleius of Madaura. Leiden: E.J. Brill, 1987.

Maass, J. G. E. Grundriss der Logik. Leipzig: Eduard Meissner, 1836.

Macfarlane, A. "Application of the Method of the Logical Spectrum to Boole's Problem." Proceedings of the American Association for Advancement of Science XXXIX (1890): 57-60. 
102 - Cogency, Journal of reasoning and argumentation

Marquand, A. “On a Logical Diagram for n Terms." Philosophical Magazine XII (1881): 266-270.

Moktefi, A. y Shin, S. “A History of Logic Diagrams." En D. M. Gabbay y J. Woods (eds.), Handbook of the History of Logic (Vol. 11). Amsterdam: Elsevier North Holland, 2012.

Moktefi, A. y Shin, S. Visual Reasoning with Diagrams. Heidelberg: Birkhauser, 2013.

Murner, T. Logica Memorativa. München: The Bavarian State Library, 1509.

Murner, T. Thomas Murner: El juego de Cartas de Lógica. Medina Delgadillo, J. (Ed.). México: Editorial Notas Universitarias, 2017.

Nakatsu, R. Diagrammatic Reasoning in AI. Hoboken: Wiley, 2010.

Nelsen, R. B. Proofs Without Words: Exercises in Visual Thinking. Washington: The Mathematical Association of America, 1993.

Newlin, William J. “A New Logic Diagram.” Journal of Philosophy, Psychology and Scientific Methods III (1906).

Newton, I. The Mathematical Principles of Natural Philosophy. Berkeley: University of California Press, 1979.

Pagnan, R. “A Diagrammatic Calculus of Syllogisms." Journal of Logic, Language and Information 21(3) (2012): 347-364.

Peirce, C. S. "Prolegomena to an Apology for Pragmaticism." Monist 16(4) (1906): 492546. Perini, L. “Truth-bearers or Truth-makers?” Spontaneous Generations: A Journal for the History and Philosophy of Science 6(1) (2012): 142-147.

Polster, B. Q.E.D.: Beauty in Mathematical Proof. Nueva York: Walker \& Co., 2004.

Roberts, D. The Existential Graphs of Charles S. Peirce. Berlín: Walter de Gruyter, 1973.

Savio, M. "AE (Aristotle-Euler) Diagrams: An Alternative Complete Method for the Categorical Syllogism." Notre Dame J. Formal Logic 39(4) (1998): 581-599. 
Shimojima, A. "Operational Constraints in Diagrammatic Reasoning." En G. Allwein y J. Barwise (eds.) Logical Reasoning with Diagrams. Nueva York: Oxford University Press, 1996.

Shin, S. The Logical Status of Diagrams. Cambridge: Cambridge University Press, 1994.

Stenning, K. Distinctions with Differences: Comparing Criteria for Distinguishing Diagrammatic from Sentential Systems. Theory and Application of Diagrams, (2000): 132-148.

Stenning, K. y Oberlander, J. “A Cognitive Theory of Graphical and Linguistic Reasoning: Logic and Implementation." Cognitive Science 19(1) (1995): 97-140.

Stevin, S. De beghinselen der weegh const. Leiden, 1586.

Stjernfelt, F. "On operational and optimal iconicity in Peirce's diagrammatology" Semiotica (186) (2011): 395-419.

Swoyer, C. "Structural representation and surrogative reasoning." Synthese 87(3) (1991): 449-508.

Tennant, N. “The Withering Away of Formal Semantics?” Mind \& Language 1(4) (1986): 302-318.

Venn, J. "On the Diagrammatic and Mechanical Representation of Propositions and Reasonings." Philosophical Magazine Series 5 10(59) (1880): 1-18. 